\title{
Stability Behavior of the Zero Solution for Nonlinear Damped Vectorial Second Order Differential Equation
}

\author{
Mohamed A. Ramadan ${ }^{1}$, Samah M. El-Kholy ${ }^{2}$ \\ ${ }^{1}$ Department of Mathematics, Faculty of Science, Minufiya University, Shebeen El-koom, Egypt \\ ${ }^{2}$ Department of Engineering Physics and Mathematics, Faculty of Engineering, Kafr El-Sheikh University, Kafr El-Sheikh, Egypt \\ Email:mramadan@eun.eg,ramadanmohamed13@yahoo.com, samahelkholy77@yahoo.com
}

Received October 7, 2012; revised November 9, 2012; accepted November 18, 2012

\begin{abstract}
In this paper, a theoretical treatment of the stability behavior of the zero solution of nonlinear damped oscillator in the vectorial case is investigated. We study the sufficient conditions for the boundedness of solution of the nonlinear damped vectorial oscillator and the conditions for the stability of the zero solution to be uniformly stable as well as asymptotically stable.
\end{abstract}

Keywords: Zero Solution; Damped Oscillator; Uniformly Stable; Asymptotically Stable

\section{Introduction}

We consider the nonlinear second order vectorial differential equation of the form

$$
\boldsymbol{x}^{\prime \prime}+2 f(t) \boldsymbol{x}^{\prime}+\beta(t) \boldsymbol{x}+\boldsymbol{g}(t, \boldsymbol{x})=0
$$

where;

$$
\begin{aligned}
& t \in R^{+}, \boldsymbol{x}=\left(x_{1}, x_{2}, \cdots, x_{n}\right) \in R^{n}, \\
& \boldsymbol{g}(t, \boldsymbol{x}): R^{+} \times R^{n} \rightarrow R^{n}, \text { and } f(t), \beta(t): R^{+} \rightarrow R .
\end{aligned}
$$

Stability problems for the second order ordinary differential equation has been intensively and widely studied [1-5]. Based on Schauder fixed point theorem T. A. Burton and T. Furumochi [2] introduced a new method to study the stability of the zero solution for Equation (1). This problem is considered also by Gheorghe Morosanu and Cristian Vladimirescu [5,6]. In [6], they used relatively classical arguments to prove the stability of the zero solution of Equation (1). While in [5], they obtained new stability results for this ordinary differential equation under more general assumptions. Their approach allows extensions to both the vector case and the case of the whole real line. In [7] the dynamics of various oscillators had been studied.

\section{The Main Results}

In the next theorem we state sufficient conations for the boundedness of the solution of Equation (1) are given.

\section{Theorem 1}

If the following hypotheses are hold:

1) $f(t) \in C^{1}\left(R^{+}\right)$and $f(t) \geq 0, \forall t \geq 0$.
2) $\beta(t) \in C^{1}\left(R^{+}\right), \quad \beta$ is decreasing and $\beta(t) \geq 1$, $\forall t \in R^{+}$.

3) $\boldsymbol{g} \in C\left(R^{+} \times R^{n}, R^{n}\right)$ and $\boldsymbol{g}$ is locally Lipschit- zian in $\left(x_{1}, \cdots, x_{n}\right)$,

4) $\boldsymbol{g}$ satisfies the following estimate $\|\boldsymbol{g}(t, \boldsymbol{x})\| \leq f(t) o(\|\boldsymbol{x}\|), \quad \forall t \in R^{+}$, where $\|\circ\|$ denotes some norm in $R^{n}$. then the solution of Equation (1) is bounded.

Proof

For the n-dimensional system, we have $\mathbf{z}=\left(x_{1}, x_{2}, \cdots, x_{n}, y_{1}, y_{2}, \cdots, y_{n}\right)^{\mathrm{T}} \in R^{m}$, where $m=2 n$.

Applying the transformation, $y_{i}=x_{i}^{\prime}+f(t) x_{i}$ and $i=1,2, \cdots, n$ Equation (1) can be converted into a first order system of differential equations of the form:

$$
\mathbf{z}^{\prime}=A(t) \mathbf{z}+B(t) \mathbf{z}+\boldsymbol{r}(t, \mathbf{z})
$$

where

$$
\text { 1) } A(t)=\left[\begin{array}{ll}
A_{1}(t) & A_{2}(t) \\
A_{3}(t) & A_{4}(t)
\end{array}\right] \text { and } B(t)=\left[\begin{array}{cc}
0 & 0 \\
B_{1}(t) & 0
\end{array}\right] \text { are }
$$

$m \times m$ matrices.

2) $A_{1}(t)=A_{4}(t)=-f(t) I, A_{2}(t)=I$, $A_{3}(t)=-\beta(t) I$, and $B_{1}(t)=\left(f^{2}(t)+f^{\prime}(t)\right) I$ are $n \times$ $n$ matrices. Note that, $0_{n \times n}$ and $I$ are the zero and the identity matrices, respectively.

3) $\boldsymbol{r}(t, \boldsymbol{x})=\left[\begin{array}{lllll}0 & \cdots & -g_{1}(t, \boldsymbol{x}) & \cdots & -g_{n}(t, \boldsymbol{x})\end{array}\right]^{\mathrm{T}}$

$$
=\left[\begin{array}{c}
0_{n \times 1} \\
-\boldsymbol{g}(t, \boldsymbol{x})
\end{array}\right]
$$

which is a $2 m \times 1$ vector.

For $i, j=1,2, \cdots, n$, let $t_{0} \geq 0$ is an arbitrary fixed and let 


$$
Z\left(t, t_{0}\right)=\left[\begin{array}{cccc}
z_{11}\left(t, t_{0}\right) & z_{12}\left(t, t_{0}\right) & \cdots & z_{1 m}\left(t, t_{0}\right) \\
z_{21}\left(t, t_{0}\right) & z_{22}\left(t, t_{0}\right) & \cdots & z_{2 m}\left(t, t_{0}\right) \\
\vdots & & & \vdots \\
Z_{m 1}\left(t, t_{0}\right) & \cdots & \cdots & z_{m m}\left(t, t_{0}\right)
\end{array}\right]=\left[\begin{array}{cc}
Z_{i, j} & Z_{i, n+j} \\
Z_{n+i, j} & Z_{n+i, n+j}
\end{array}\right]
$$

be a fundamental matrix solution to the linear system:

$$
\mathbf{z}^{\prime}=A(t) \mathbf{z}
$$

which equal to the identity matrix for $t=t_{0}$.

Consider $\mathbf{z}_{0} \neq 0$ with $\left\|\mathbf{z}_{0}\right\|$ small enough, $t_{0} \geq 0$ and let us denote by $\mathbf{z}\left(t, t_{0}, \mathbf{z}_{0}\right)$ the unique solution of Equation (2) which equal to $\vec{z}_{0}$ at $t=t_{0}$. By hypotheses (1) and (2), $\mathbf{z}\left(t, t_{0}, \mathbf{z}_{0}\right)$ is defined on a maximal right interval, $\left[t_{0}, l\right)$, and satisfies the following integral equation:

$$
\mathbf{z}\left(t, t_{0}, \mathbf{z}_{0}\right)=Z\left(t, t_{0}\right) \mathbf{z}_{0}+\int_{t_{0}}^{t} Z\left(t, t_{0}\right) Z^{-1}\left(s, t_{0}\right)\left[B(s) \mathbf{z}\left(s, t_{0}, \mathbf{z}_{0}\right)+\boldsymbol{r}\left(s, \mathbf{z}\left(s, t_{0}, \mathbf{z}_{0}\right)\right)\right] \mathrm{d} s
$$

This gives us the following integral inequality:

$$
\left\|\mathbf{z}\left(t, t_{0}, \mathbf{z}_{0}\right)\right\| \leq\left\|Z\left(t, t_{0}\right) \mathbf{z}_{0}\right\|+\int_{t_{0}}^{t}\left\|Z\left(t, t_{0}\right) Z^{-1}\left(s, t_{0}\right) \mathrm{e}\right\|\left[\|B(s)\|\left\|\mathbf{z}\left(s, t_{0}, \mathbf{z}_{0}\right)\right\|+\left\|\boldsymbol{r}\left(s, \mathbf{z}\left(s, t_{0}, \mathbf{z}_{0}\right)\right)\right\|\right] \mathrm{d} s
$$

where $e=\left[\begin{array}{lllll}0 & \cdots & 1 & 0 & \cdots\end{array}\right]^{\mathrm{T}}$.

Equations (3) and (4) give us the following differential equations:

$$
\begin{gathered}
z_{i, j}^{\prime}=-f(t) z_{i, j}+z_{n+i, j} \\
z_{n+i, j}^{\prime}=-\beta(t) z_{i, j}-f(t) z_{n+i, j} \\
z_{i, n+j}^{\prime}=-f(t) z_{i, n+j}+z_{n+i, n+j} \\
z_{n+i, n+j}^{\prime}=-\beta(t) z_{i, n+j}-f(t) z_{n+i, n+j}
\end{gathered}
$$

for $i, j=1,2, \cdots, n$.

Since $\beta(t)$ is a decreasing function, so Equations (6) and (7) lead to:

$$
\begin{aligned}
\frac{1}{2}\left[\beta(t) z_{i, j}^{2}+z_{n+i, j}^{2}\right]^{\prime} \leq-f(t) & {\left[\beta(t) z_{i, j}^{2}+z_{n+i, j}^{2}\right] \quad \begin{array}{l}
\text { For } \quad \mathbf{z}_{0}=\left(x_{01}, x_{02}, \cdots, x_{0 n}, y_{01}, y_{02},\right. \\
\text { have: }
\end{array} } \\
\left\|Z\left(t, t_{0}\right) \mathbf{z}_{0}\right\| & =\left\|\left[\begin{array}{cc}
Z_{i, j} & Z_{i, n+j} \\
Z_{n+i, j} & Z_{n+i, n+j}
\end{array}\right]\left[\begin{array}{l}
\boldsymbol{x}_{0} \\
\boldsymbol{y}_{0}
\end{array}\right]\right\|=\left\|\left[\begin{array}{c}
Z_{i, j} \boldsymbol{x}_{0}+Z_{i, n+j} \boldsymbol{y}_{0} \\
Z_{n+i, j} \boldsymbol{x}_{0}+Z_{n+i, n+j} \boldsymbol{y}_{0}
\end{array}\right]\right\| \\
& =\sqrt{\sum_{i=1}^{n}\left[\left[\sum_{j=1}^{n}\left(z_{i, j} x_{0 j}+z_{i, n+j} y_{0 j}\right)\right]^{2}+\left[\sum_{j=1}^{n}\left(z_{n+i, j} x_{0 j}+Z_{n+i, n+j} y_{0 j}\right)\right]^{2}\right]}
\end{aligned}
$$
and (9) the following: in $R^{m}$.

By the same way we can obtain from Equations (8)

$$
\beta(t) z_{i, n+j}^{2}+z_{n+i, n+j}^{2} \leq \mathrm{e}^{-2 \int^{t} f(u) \mathrm{d} u}
$$

For $\mathbf{z}=\left(x_{1}, x_{2}, \cdots, x_{n}, y_{1}, y_{2}, \cdots, y_{n}\right)^{\mathrm{T}} \in R^{m}$, consider the norm $\|z\|=\sqrt{\sum_{i=1}^{m} z_{i}^{2}}$, where $\|\cdot\|$ is the norm defined

For $\mathbf{z}_{0}=\left(x_{01}, x_{02}, \cdots, x_{0 n}, y_{01}, y_{02}, \cdots, y_{0 n}\right)^{\mathrm{T}} \in R^{m} \quad$ we

Using Shwartz inequality, Minkowski inequality [8] and suitable assumptions lead to:

$$
\left\|Z\left(t, t_{0}\right) \mathbf{z}_{0}\right\| \leq n \sqrt{3 \beta\left(t_{0}\right)+1} \mathrm{e}^{-2 \int_{0}^{t} f(u) \mathrm{d} u}\left\|\mathbf{z}_{0}\right\|
$$

We have also:

$$
\begin{aligned}
& \forall t \geq s \geq t_{0} \geq 0 \\
& Z\left(t, t_{0}\right) Z^{-1}\left(s, t_{0}\right) \mathrm{e} \\
& =\left[\begin{array}{llll}
\eta_{1}\left(t, s, t_{0}\right) & \eta_{2}\left(t, s, t_{0}\right) & \cdots & \eta_{m}\left(t, s, t_{0}\right)
\end{array}\right]^{\mathrm{T}}
\end{aligned}
$$

satisfies (4), then we get:

$$
\begin{aligned}
& \left\|Z\left(t, t_{0}\right) Z^{-1}\left(s, t_{0}\right) \mathrm{e}\right\| \\
& =\sqrt{\sum_{i=1}^{m} \eta_{i}^{2}\left(t, s, t_{0}\right)} \\
& \leq \sqrt{\sum_{i=1}^{n}\left(\beta(t) \eta_{i}^{2}\left(t, s, t_{0}\right)+\eta_{i+n}^{2}\left(t, s, t_{0}\right)\right)}
\end{aligned}
$$

Since $\beta(t)$ is a decreasing function of $t$, we get: 


$$
\left[\beta(t) \eta_{i}^{2}\left(t, s, t_{0}\right)+\eta_{n+i}^{2}\left(t, s, t_{0}\right)\right] \leq\left[\beta(s) \eta_{i}^{2}\left(s, s, t_{0}\right)+\eta_{n+i}^{2}\left(s, s, t_{0}\right)\right] \mathrm{e}^{-2 \int_{s}^{t} f(u) \mathrm{d} u}
$$

So,

$$
\left\|Z\left(t, t_{0}\right) Z^{-1}\left(s, t_{0}\right) e\right\| \leq \sqrt{n \beta\left(t_{0}\right)} e^{-\int_{s}^{t} f(u) \mathrm{d} u}
$$

As mentioned the system satisfies the integral inequality (5), then inequalities (12) and (13) give

$$
\begin{gathered}
\left\|\mathbf{z}\left(t, t_{0}, \mathbf{z}_{0}\right)\right\| \leq\left\|Z\left(t, t_{0}\right) \mathbf{z}_{0}\right\|+\int_{t_{0}}^{t}\left\|Z\left(t, t_{0}\right) Z^{-1}\left(s, t_{0}\right) \mathrm{e}\right\|\left[\|B(s)\|\left\|\mathbf{z}\left(s, t_{0}, \mathbf{z}_{0}\right)\right\|+\left\|\boldsymbol{r}\left(s, \mathbf{z}\left(s, t_{0}, \mathbf{z}_{0}\right)\right)\right\|\right] \mathrm{d} s \\
\left\|\mathbf{z}\left(t, t_{0}, \mathbf{z}_{0}\right)\right\| \leq n \sqrt{3 \beta\left(t_{0}\right)+1} \mathrm{e}^{-\int_{0}^{t} f(u) \mathrm{d} u}\left\|\mathbf{z}_{0}\right\|+\int_{t_{0}}^{t} \sqrt{n \beta\left(t_{0}\right)} \mathrm{e}^{-\int_{t s}^{t} f(u) \mathrm{d} u}\left[n \mid f^{2}(s)+f^{\prime}(s)\left\|\mathbf{z}\left(s, t_{0}, \mathbf{z}_{0}\right)\right\|+\|\boldsymbol{g}(s, \boldsymbol{x})\|\right] \mathrm{d} s
\end{gathered}
$$

For all $t$ we can replace $\boldsymbol{g}$ by another function say $\boldsymbol{g}$ defined as follows. By hypothesis (4) it follows that there exists a $\rho>0$ such that if $\|x\|<\rho$, then

$$
\|\boldsymbol{g}(t, \boldsymbol{x})\| \leq \theta f(t) o(\|\boldsymbol{x}\|)
$$

We defined the function $\tilde{\boldsymbol{g}}: R^{+} \times R^{n} \rightarrow R^{n}$ by:

$$
\tilde{\boldsymbol{g}}(t, \boldsymbol{x})= \begin{cases}g(t, \boldsymbol{x}) & \text { if }\|\boldsymbol{x}\|<\rho \\ g(t, \tilde{\boldsymbol{x}}) & \text { if }\|\boldsymbol{x}\| \geq \rho\end{cases}
$$

and we have $\|\tilde{\boldsymbol{x}}\|=\rho, \forall t \geq 0$.

It is clear that for every $(t, x) \in R^{+} \times R^{n}$

$$
\|\boldsymbol{g}(t, \boldsymbol{x})\| \leq \theta f(t) o(\|\boldsymbol{x}\|)
$$

$\tilde{\boldsymbol{g}}$ is of class $C\left(R^{+} \times R^{n}\right)$ and is locally Lipschitzian in $\left(x_{1}, x_{2}, \cdots, x_{n}\right)$. So, we will admit from now that the original function $\boldsymbol{g}$ satisfies all the properties of the $\tilde{\boldsymbol{g}}$. Since $f \in C^{1}\left(R^{+}\right)$we get from inequality (14) that:

$$
\begin{aligned}
\left\|\mathbf{z}\left(t, t_{0}, \mathbf{z}_{0}\right)\right\| \leq & n \sqrt{3 \beta\left(t_{0}\right)+1} \mathrm{e}^{-\int^{t_{0}} f(u) \mathrm{d} u}\left\|\mathbf{z}_{0}\right\| \\
& +D \int_{t_{0}}^{t}\left\|\mathbf{z}\left(s, t_{0}, \mathbf{z}_{0}\right)\right\| \mathrm{d} s
\end{aligned}
$$

$\forall t \in\left[t_{0}, l\right)$, with positive constant $\mathrm{D}[9,10]$. This gives us the following

$$
\left\|\mathbf{z}\left(t, t_{0}, \mathbf{z}_{0}\right)\right\| \leq n \sqrt{3 \beta\left(t_{0}\right)+1} e^{D h}\left\|\mathbf{z}_{0}\right\|, \forall t \in\left[t_{0}, l\right)
$$

Thus $\left\|\mathbf{z}\left(t, t_{0}, \mathbf{z}_{0}\right)\right\|$ as well as $\left\|\mathbf{z}^{\prime}\left(t, t_{0}, \mathbf{z}_{0}\right)\right\|$ are bounded on $\left[t_{0}, l\right)$ and so $\left\|\mathbf{z}\left(t, t_{0}, \mathbf{z}_{0}\right)\right\|$ can be extended to the right of $l$. This contradicts the maximality of $l$. This means that the solution of Equation (1) is bounded. The proof of theorem 1 is complete.

\section{Theorem 2}

If the hypotheses of theorem 1 are hold and the following assumption is satisfied:

1) There exist two constants $h, k \geq 0$ such that:

$$
\left|f^{\prime}(t)+f^{2}(t)\right| \leq k f(t) \quad \forall t \in[h, \infty)
$$

then the zero solution of Equation (1) is uniformly stable solution.

If in addition

2) $\int_{0}^{\infty} f(t) \mathrm{d} t=\infty$ holds, then the zero solution of Equation (1) is asymptotically stable.

\section{Proof}

Our stability question is reduced to the stability of the zero solution $\mathbf{z}(t)=0$ to the system (2). The interval of $t$ will be divided into two intervals. In the first interval we have $t \in\left[t_{0}, h\right)$ and in the second interval $t \in[h, \infty)$. Firstly, with $l<h$ and since the hypotheses of theorem 1 are hold, and then the solution $\mathbf{z}\left(t, t_{0}, \mathbf{z}_{0}\right)$ of Equation (1) is defined on a maximal right interval $\left[t_{0}, l\right)$. It is proved in theorem 1 that the solution is bounded and $\left\|\mathbf{z}\left(t, t_{0}, \mathbf{z}_{0}\right)\right\|$ can be extended to the right of $l$. Therefore, $\left\|\mathbf{z}\left(t, t_{0}, \mathbf{z}_{0}\right)\right\|$ exists on $\left[t_{0}, l\right)$ with $l>h$.

Assume $h<l<\infty$. We are going to find an estimate for $\left\|\mathbf{z}\left(t, t_{0}, \mathbf{z}_{0}\right)\right\|$ on the interval $[h, l)$. From hypothesis (1) of theorem 2, we have for $\forall t \in[h, l)$ :

$$
\begin{gathered}
\left\|\mathbf{z}\left(t, t_{0}, \mathbf{z}_{0}\right)\right\| \leq n \sqrt{3 \beta(h)+1} \mathrm{e}^{-\int^{t} f(u) \mathrm{d} u}\left\|\mathbf{z}_{0}\left(h, t_{0}, \mathbf{z}_{0}\right)\right\|+\int_{h}^{t} \sqrt{n \beta(h)} \mathrm{e}^{-\int^{t} f(u) \mathrm{d} u}\left(n k \mid f(s)\left\|\mathbf{z}\left(s, t_{0}, \mathbf{z}_{0}\right)\right\|+\|g(s, \boldsymbol{x})\|\right) \mathrm{d} s \\
\left\|\mathbf{z}\left(t, t_{0}, \mathbf{z}_{0}\right)\right\| \leq n \sqrt{3 \beta(h)+1} \mathrm{e}^{-\int^{t} f(u) \mathrm{d} u}\left\|\mathbf{z}_{0}\left(h, t_{0}, \mathbf{z}_{0}\right)\right\|+\int_{h}^{t} \sqrt{n \beta(h)} \mathrm{e}^{-\int^{t} f(u) \mathrm{d} u}\left(n k\left|f(s)\left\|\mathbf{z}\left(s, t_{0}, \mathbf{z}_{0}\right)\right\|+\theta\right| f(s)\left\|\mathbf{x}\left(s, t_{0}, \mathbf{z}_{0}\right)\right\|\right) \mathrm{d} s
\end{gathered}
$$


with

$$
\begin{aligned}
& \theta \in\left(0, \frac{1}{\sqrt{n \beta(h)}}-n k\right) \\
& \left\|\mathbf{z}\left(t, t_{0}, \mathbf{z}_{0}\right)\right\| \\
& \leq n \sqrt{3 \beta(h)+1} \mathrm{e}^{-\int_{h}^{t} f(u) \mathrm{d} u}\left\|\mathbf{z}_{0}\left(h, t_{0}, \mathbf{z}_{0}\right)\right\| \\
& +\int_{h}^{t} \sqrt{n \beta(h)} \mathrm{e}^{-\int^{t} f(u) \mathrm{d} u}\left([n k+\theta] \mid f(s)\left\|\mathbf{z}\left(s, t_{0}, \mathbf{z}_{0}\right)\right\|\right) \mathrm{d} s
\end{aligned}
$$$$
v(t)=\sqrt{3 \beta(h)+1} \mathrm{e}^{-\iint^{t} f(u) \mathrm{d} u}\left\|\mathbf{z}_{0}\left(h, t_{0}, \mathbf{z}_{0}\right)\right\|
$$$$
+\int_{h}^{t} \sqrt{n \beta(h)} \mathrm{e}^{-\int^{t} f(u) \mathrm{d} u}[n k+\theta] \mid f(s)\left\|\boldsymbol{z}\left(s, t_{0}, \mathbf{z}_{0}\right)\right\| \mathrm{d} s
$$

So

$$
v^{\prime}(t) \leq[\sqrt{n \beta(h)}(n k+\theta)-1] f(t) v(t) \quad \forall t \in[h, l) .
$$

By integration we get:

$$
\begin{aligned}
& \left\|\boldsymbol{z}\left(t, t_{0}, \mathbf{z}_{0}\right)\right\| \leq v(t) \leq v(h) \mathrm{e}^{[\sqrt{n \beta(h)}(n k+\theta)-1]]_{h}^{t} f(u) \mathrm{d} u}, \\
& \forall t \in[h, l)
\end{aligned}
$$

with $v(h)=n \sqrt{3 \beta(h)+1}\left\|\mathbf{z}\left(h, t_{0}, \mathbf{z}_{0}\right)\right\|$

From inequality (18) we can see that $\left\|\mathbf{z}\left(t, t_{0}, \mathbf{z}_{0}\right)\right\|$ is bounded since $\left\|\mathbf{z}^{\prime}\left(t, t_{0}, \mathbf{z}_{0}\right)\right\|$ is also bounded, it follows that $l=\infty$. For $\varepsilon>0$ we can get:

$$
\delta(\varepsilon)=\frac{\varepsilon \mathrm{e}^{-D h}}{n^{2}(\sqrt{1+3 \beta(0)})(\sqrt{1+3 \beta(h)})}
$$

From (15) it follows that $\left\|\mathbf{z}\left(t, t_{0}, \mathbf{z}_{0}\right)\right\| \leq \frac{\varepsilon}{n \sqrt{1+3 \beta(h)}}$ for all $t \in\left[t_{0}, h\right]$ provided that $\left\|\mathbf{z}_{0}\right\|<\delta$. Therefore, from (16) and (18), we get $\left\|\boldsymbol{z}\left(t, t_{0}, \mathbf{z}_{0}\right)\right\| \leq v(h)<\varepsilon$ for all $t \geq h$. So, if $0 \leq t_{0} \leq h$, then the solution $\mathbf{z}\left(t, t_{0}, \mathbf{z}_{0}\right)$ starting from any point $\mathbf{z}_{0}$, with $\left\|\mathbf{z}_{0}\right\|<\delta$, exists on $\left[t_{0}, \infty\right)$ and satisfies $\left\|\mathbf{z}\left(t, t_{0}, \mathbf{z}_{0}\right)\right\|<\varepsilon$ for all $t \geq t_{0}$. If $t_{0} \geq h$, then analogously we obtain that $l=\infty$ and:

$$
\begin{aligned}
& \left\|\mathbf{z}\left(t, t_{0}, \mathbf{z}_{0}\right)\right\| \leq n \sqrt{3 \beta\left(t_{0}\right)+1}\left\|\mathbf{z}_{0}\right\| \mathrm{e}^{[\sqrt{n \beta(h)}(n k+\theta)-1] \int_{t_{0}}^{t} f(u) \mathrm{d} u}, \\
& \forall t \in[h, \infty)
\end{aligned}
$$

Therefore, with the same $\delta$ as before, $\left\|\mathbf{z}_{0}\right\|<\delta$ implies again $\left\|\mathbf{z}\left(t, t_{0}, \mathbf{z}_{0}\right)\right\|<\varepsilon$ for all $t \geq t_{0}$. Hence the zero solution is uniformly stable.

If in addition

$$
\int_{0}^{\infty} f(t) \mathrm{d} t=\infty
$$

is satisfied, then by (16), (18) and (19) it follows that the zero solution to (1) is asymptotically stable. The proof of theorem 2 is complete.

\section{Examples}

We confirm the results of the introduced theorems by considering two numerical examples for which the functions $f(t), \beta(t)$ and $\boldsymbol{g}(t)$ satisfy theorems assumptions.

For the system

$$
\boldsymbol{x}^{\prime \prime}+2 f(t) \boldsymbol{x}^{\prime}+\beta(t) \boldsymbol{x}+\boldsymbol{g}(t, \boldsymbol{x})=0,
$$

where $\vec{x}=\left(x_{1}, x_{2}\right)$.

\section{Example 1}

$f(t)=\frac{t}{t^{2}+1}, \quad \beta(t)=1+1 / t, \quad g_{1}\left(t, x_{1}, x_{2}\right)=\frac{x_{1} x_{2} t}{t^{2}+1}$ and $g_{2}\left(t, x_{1}, x_{2}\right)=\frac{\left(x_{1}^{2}+x_{2}^{2}\right) t}{t^{2}+1}$.

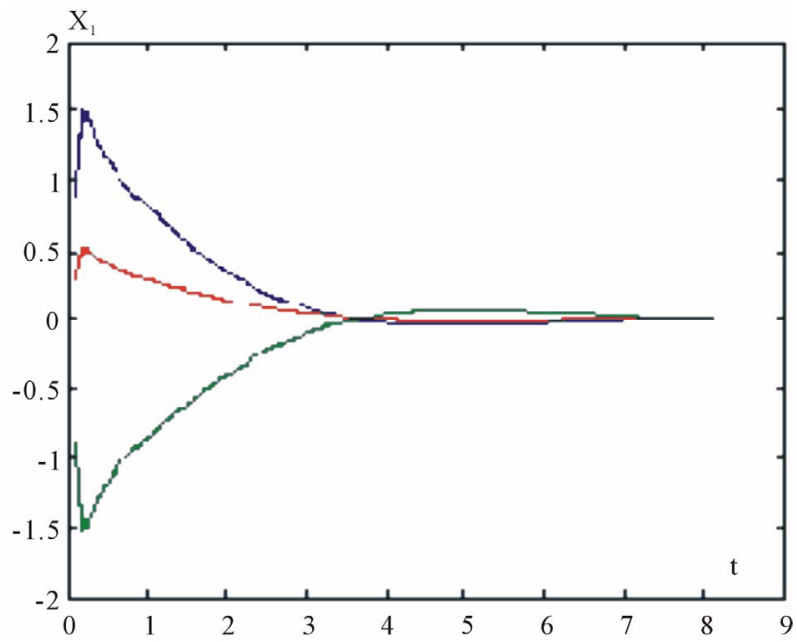

(a)

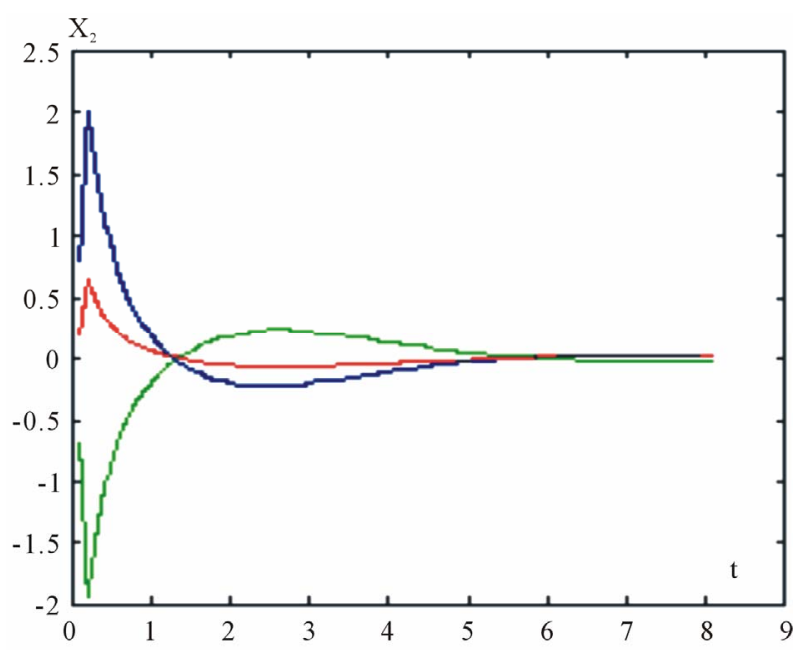

(b)

Figure 1. Numerical solutions if Example 1, $x_{1}$ component (a) and $x_{2}$ component (b). 


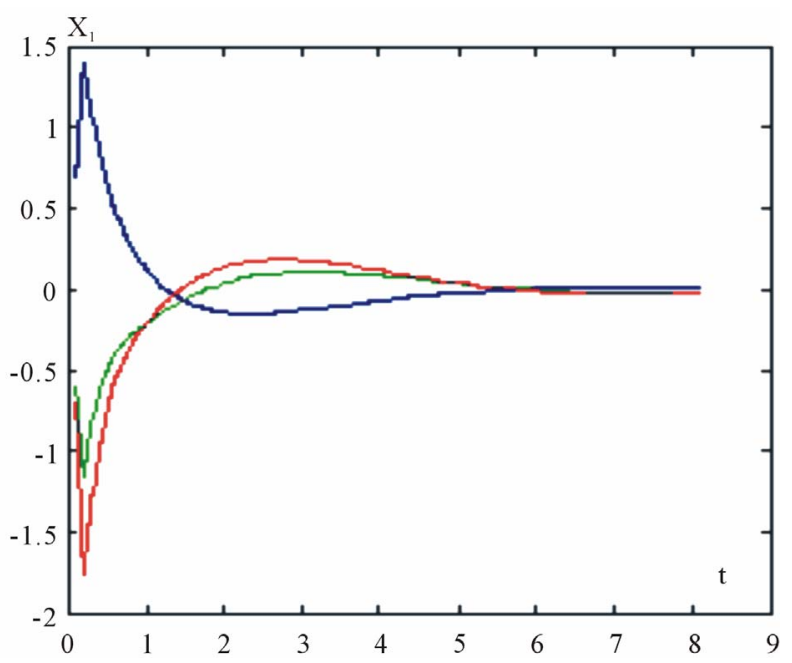

(a)

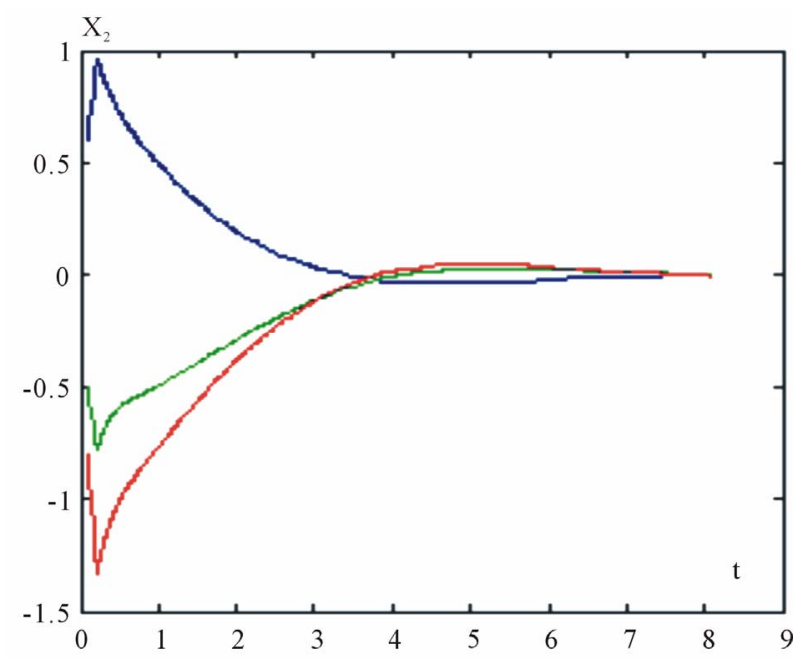

(b)

Figure 2. Numerical solutions if example 2, $x_{1}$ component (a) and $x_{2}$ component (b).

\section{Example 2}

$$
\begin{aligned}
& f(t)=\frac{1}{t^{2}}, \quad \beta(t)=1+\mathrm{e}^{-t^{2}}, \quad g_{1}\left(t, x_{1}, x_{2}\right)=\frac{x_{1} x_{2}}{t^{2}} \text { and } \\
& g_{2}\left(t, x_{1}, x_{2}\right)=\frac{\left(x_{1}^{2}+x_{2}^{2}\right)}{t^{2}} .
\end{aligned}
$$

We solved these two examples numerically using fourth order Runge-Kutta method. The results of example 1 are drawn as shown in Figure $\mathbf{1}$ and the results of exam- ple 2 are drawn as shown in Figure 2. The curves are drawn for different initial values of $\boldsymbol{x}_{0}$.

Figures $\mathbf{1}$ and $\mathbf{2}$ demonstrate that, as the time increases all the components of the solutions tends to zero. This means, that the solutions are asymptotically stable. Which verify the rightness of our proved theorems.

\section{Conclusion}

We introduced two theorems which provide the sufficient conditions for the boundedness of solution of the nonlinear damped vectorial oscillator and the conditions for the stability of the zero solution to be uniformly stable as well as asymptotically stable. We verified our theoretical results by solving two examples satisfying the assumptions of the two proved theorems.

\section{REFERENCES}

[1] T. A. Burton and T. Furumochi, "A Note on Stability by Schauder's Theorem," Funkcialaj Ekvacioj, Vol. 44, No. 1, 2001, pp. 73-82.

[2] V. A. Coppel, "Stability and Asymptotic Behavior of Differential Equations," D. C. Heath and Company, Lexington, 1965.

[3] D. Grossman, "Introduction to Differential Equations with Boundary Value Problems," Wiley-Interscience, John Wiley \& Sons, Inc., Hoboken, 1987.

[4] J. Hale, "Ordinary Differential Equations," Wiley Interscience, John Wiley \& Sons, Inc., Hoboken, 1969.

[5] G. H. Moroşanu and C. Vladimirescu, "Stability for a Nonlinear Second Order ODE," Funkcialaj Ekvacioj, Vol. 48, No. 1, 2005, pp. 49-56. doi:10.1619/fesi.48.49

[6] G. H. Moroşanu and C. Vladimirescu, "Stability for a Damped Nonlinear Oscillator," Nonlinear Analysis, Vol. 60, No. 2, 2005, pp. 303-310.

[7] J. Awrejcewicz, "Classical Mechanics. Dynamics," Springer-Verlag, New York, 2012,

[8] R. F. Curtain and A. J. Pritchard, "Functional Analysis in Modern Applied Mathematics,” Academic Press Inc. Ltd., London, 1977.

[9] R. Bellman, "Stability Theory of Differential Equations," Dover Publications Inc., Mineola, 1953.

[10] V. Lakshmikantham and S. Leela, "Differential and Integral Inequalities. Theory and Applications," Academic Press, New York and London, 1969. 